\title{
COSMIC-RAY-MEDIATED FORMATION OF BENZENE ON THE SURFACE OF SATURN'S MOON TITAN
}

\author{
Li Zhou ${ }^{1,7}$, Weijun Zheng ${ }^{1,8}$, Ralf I. Kaiser ${ }^{1,9}$, Alexander Landera ${ }^{2}$, Alexander M. Mebel ${ }^{2}$, Mao-Chang Liang $^{3,4,5}$, \\ AND YUK L. YUNG ${ }^{6}$ \\ ${ }^{1}$ Department of Chemistry, University of Hawaii at Manoa, Honolulu, HI, USA \\ ${ }^{2}$ Department of Chemistry and Biochemistry, Florida International University, Miami, FL, USA \\ ${ }^{3}$ Research Center for Environmental Changes, Academia Sinica, Taipei, Taiwan \\ ${ }^{4}$ Graduate Institute of Astronomy, National Central University, Jhongli, Taiwan \\ ${ }^{5}$ Institute of Astronomy and Astrophysics, Academia Sinica, Taipei, Taiwan \\ ${ }^{6}$ Division of Geological and Planetary Sciences, Caltech, Pasadena, CA, USA \\ Received 2009 November 11; accepted 2010 April 11; published 2010 July 14
}

\begin{abstract}
The aromatic benzene molecule $\left(\mathrm{C}_{6} \mathrm{H}_{6}\right)$ - a central building block of polycyclic aromatic hydrocarbon molecules-is of crucial importance for the understanding of the organic chemistry of Saturn's largest moon, Titan. Here, we show via laboratory experiments and electronic structure calculations that the benzene molecule can be formed on Titan's surface in situ via non-equilibrium chemistry by cosmic-ray processing of low-temperature acetylene $\left(\mathrm{C}_{2} \mathrm{H}_{2}\right)$ ices. The actual yield of benzene depends strongly on the surface coverage. We suggest that the cosmic-ray-mediated chemistry on Titan's surface could be the dominant source of benzene, i.e., a factor of at least two orders of magnitude higher compared to previously modeled precipitation rates, in those regions of the surface which have a high surface coverage of acetylene.
\end{abstract}

Key words: astrochemistry - comets: general - methods: laboratory - molecular processes - planets and satellites: individual (Titan)

Online-only material: color figures

\section{INTRODUCTION}

Titan is the largest satellite of Saturn and the only moon in our solar system known to have a dense, planet-like atmosphere with pressures of 1.4 bar and temperatures of $94 \mathrm{~K}$ at the surface (Lunine \& Lorenz 2009). Titan's atmosphere is dominated by molecular nitrogen $\left(\mathrm{N}_{2} ; 98.4 \%\right)$ with the remaining $1.6 \%$ composed mainly of methane $\left(\mathrm{CH}_{4}\right)$ and trace hydrocarbon gases (acetylene $\left(\mathrm{C}_{2} \mathrm{H}_{2}\right)$, ethylene $\left(\mathrm{C}_{2} \mathrm{H}_{4}\right)$, ethane $\left(\mathrm{C}_{2} \mathrm{H}_{6}\right)$, methylacetylene $\left(\mathrm{CH}_{3} \mathrm{CCH}\right)$, propane $\left(\mathrm{C}_{3} \mathrm{H}_{8}\right)$, diacetylene $\left(\mathrm{C}_{4} \mathrm{H}_{2}\right)$, benzene $\left.\left(\mathrm{C}_{6} \mathrm{H}_{6}\right)\right)$, nitriles (hydrogen cyanide $(\mathrm{HCN})$, cyanoacetylene ( $\mathrm{HCCCN})$, cyanogen $\left(\mathrm{C}_{2} \mathrm{~N}_{2}\right)$ ), and oxygen-bearing molecules (carbon dioxide $\left(\mathrm{CO}_{2}\right)$, carbon monoxide $(\mathrm{CO})$, water $\left(\mathrm{H}_{2} \mathrm{O}\right)$ ) (Coustenis et al. 2007). Solar photons drive a rich photochemistry leading eventually to the formation of Titan's orange-brownish organic haze layers (Liang et al. 2007; Lebonnois 2005; Lavvas et al. 2008). These layers contain predominant antigreenhouse species and control Titan's climate including wind and rain (Flasar 2006; Raulin 2008; Griffith et al. 2000). Here, polycyclic aromatic hydrocarbons (PAHs), organic molecules with fused benzene rings, are considered key components of Titan's haze and potential constituents of the organic material on Titan's surface (Wilson \& Atreya 2004; Imanaka et al. 2004; Coustenis \& Hirtzig 2009). However, the underlying formation routes of even the simplest building block of PAHs - the aromatic benzene molecule $\left(\mathrm{C}_{6} \mathrm{H}_{6}\right)$ - have not been understood to date.

The benzene molecule itself was first observed in Titan's atmosphere by Coustenis et al. (2003) utilizing data of the

\footnotetext{
7 Present address: Chemistry Department, Nanchang University, Nanchang 330031, China.

8 Present address: Beijing National Laboratory for Molecular Sciences, State Key Laboratory of Molecular Reaction Dynamics, Institute of Chemistry, Chinese Academy of Sciences, Beijing 100190, China.

9 Author to whom any correspondence should be addressed.
}

Infrared Space Observatory at $674 \mathrm{~cm}^{-1}$ at molar fractions of $4 \times 10^{-10}$ averaged over the disk. Data from the Composite Infrared Spectrometer aboard the Cassini spacecraft presented a firm detection of benzene at mixing ratios that vary from $3.5 \times$ $10^{-9}$ at $70^{\circ} \mathrm{N}$ to about $1 \times 10^{-10}$ at $70^{\circ} \mathrm{S}$. An analysis of the data from the Cassini Mass Spectrometer (INMS) suggested that in Titan's ionosphere, benzene could be formed via ion-molecule reactions at fluxes of $10^{7} \mathrm{~cm}^{-2} \mathrm{~s}^{-1}$ (Vuitton et al. 2008), which are of the same order of magnitude as neutral-neutral reactions involving propargyl radicals (Wilson et al. 2003). Most noticeably, the Huygens probe, launched from the Cassini spacecraft, conducted the first in situ analysis of the chemical composition of Titan's surface, reporting benzene qualitatively via Gas Chromatograph Mass Spectrometer measurements at the Huygens landing site (Niemann et al. 2005). However, the origin of benzene on Titan's surface is unclear. Is the surface concentration of benzene the result of a downward transport of benzene formed in the higher atmosphere or is heterogeneous surface chemistry involved?

Considering the low temperature of Titan's surface of 94 $\mathrm{K}$, classical thermal chemistry of closed shell species in the solid state does not occur, since activation energies prohibit any chemical reaction. However, Sagan \& Thompson (1984) outlined that energetic cosmic-ray particles can penetrate deep into the lower atmospheric layers; these energetic particles could incorporate part of their kinetic energy into the chemical reaction and thus process simple organics in Titan's lower atmosphere. Note, however, that the authors did not calculate the energy flux deposited directly on Titan's surface. In a more recent study, Molina-Cuberosa et al. (1999) derived an energy deposition on Titan's surface of $4.5 \times 10^{9} \mathrm{eV} \mathrm{cm}^{-2} \mathrm{~s}^{-1}$. This process develops into an electromagnetic cascade of electrons and photons known as a cascade shower. Conversely, there has been no conclusion as to what degree these processes actually lead to the formation 
of molecules such as benzene on Titan's surface. Therefore, an experimental study of the cosmic-ray-mediated synthesis of complex molecules on Titan's surface is highly warranted. Here, we report on laboratory simulation experiments on the formation of benzene $\left(\mathrm{C}_{6} \mathrm{H}_{6}\right)$ on Titan's surface via the processing of low-temperature acetylene $\left(\mathrm{C}_{2} \mathrm{H}_{2}\right)$ ices by energetic electrons as generated in the track of Galactic cosmic-ray particles. The experiments are combined with electronic structure calculations to propose feasible reaction pathways to synthesize benzene via excited-state chemistry. We also estimate-incorporating heterogeneous, cosmic-ray-triggered surface chemistry-the surface production of benzene under realistic, planetary-like conditions. Finally, we expand on our findings and propose potential pathways to the formation of more complex, heterocyclic aromatic systems. It should be stressed that no single simulation experiment on the interaction of cosmic-ray particles with frozen organics can mimic the complexity of Titan's environment, for example, a wide energy range of electrons generated in the track of cosmic-ray particles and the complex chemical composition of Titan's surface, simultaneously. An understanding of these processes must be based on simulation experiments which first involve relatively simple model systems under controlled conditions, before extending the simulation conditions to more complex systems. In this case, we utilized acetylene as a prototype 'building block' to form aromatic molecules such as benzene. Likewise, energetic electrons with kinetic energies of $5 \mathrm{keV}$ were selected since they have a linear energy transfer similar to high-energy galactic cosmic-ray particles and transfer their kinetic energy exclusively via inelastic processes, but not via nuclear interaction.

\section{EXPERIMENTAL DETAILS}

Acetylene ices of $286 \pm 10 \mathrm{~nm}$ thickness were prepared at temperatures of $10 \mathrm{~K}, 30 \mathrm{~K}, 50 \mathrm{~K}$, and $67 \mathrm{~K}$ and irradiated with energetic electrons with kinetic energies of $5 \mathrm{keV}$ at nominal fluxes of $4.9 \times 10^{11} \mathrm{~cm}^{-2} \mathrm{~s}^{-1}$ for $1 \mathrm{hr}$ (Bennett et al. 2005). The irradiated samples were kept isothermal for $1 \mathrm{hr}$ and were then warmed up at a rate of $0.5 \mathrm{~K}_{\text {minute }}{ }^{-1}$ to $300 \mathrm{~K}$; this allowed the reactants and product molecules to sublime. During the experiment, the chemical processing of the acetylene ices was followed on line and in situ via a Fourier transform infrared spectrometer (Nicolet 6700) in the range of $3500-500 \mathrm{~cm}^{-1}$. The gas phase was sampled simultaneously with a quadrupole mass spectrometer from 1 to $200 \mathrm{amu}$ after electron impact ionization of the molecules with $80 \mathrm{eV}$ electrons (Balzer QMG 420). Control experiments were conducted with deuterated and ${ }^{13} \mathrm{C}$-substituted acetylene samples to confirm the infrared and mass spectroscopic assignments. We would like to comment on the computations of the column density. Here, the column densities are calculated via a modified Lambert-Beer law in units of molecules per $\mathrm{cm}^{2}$ utilizing the absorptions and integral absorption coefficients for vinylacetylene $\left(1599 \mathrm{~cm}^{-1}, 1.4 \times\right.$

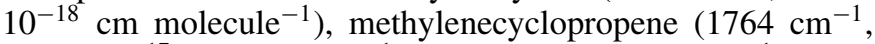
$3.2 \times 10^{-17} \mathrm{~cm}$ molecule $\left.^{-1}\right)$, and benzene $\left(1480 \mathrm{~cm}^{-1}, 1.5 \times\right.$ $10^{-18} \mathrm{~cm}$ molecule $\left.{ }^{-1}\right)$. By dividing the column density by the sample thickness in centimeters, the concentration, i.e., the molecules per $\mathrm{cm}^{3}$, can be calculated. For the quantification of the newly formed molecules and an incorporation of these data into models, the column density has the advantage that a simple multiplication with the sample area yields the total numbers of molecules formed during the irradiation at a defined time.

\section{COMPUTATIONS}

The electronic structure calculations were conducted at a level of theory high enough to predict relative energies of all local minima, transition states, and products of the reaction of acetylene to a precision of about $5 \mathrm{~kJ} \mathrm{~mol}^{-1}$. Stationary points were optimized at the hybrid density functional B3LYP level with the $6-311 \mathrm{G}^{* *}$ basis set. Vibrational frequencies were calculated using the same B3LYP/6-311G** method and were used to compute zero-point vibrational energy (ZPE) corrections and reaction rate constants without scaling. Relative energies of various species were refined employing the coupled cluster CCSD(T) method (Becke 1993; Lee et al. 1988; Purvis \& Bartlett 1982; Scuseria et al. 1988; Scuseria \& Schaefer 1989; Pople et al. 1987) with extrapolation to the complete basis set limit and including ZPEs obtained by B3LYP calculations.

\section{EXPERIMENTAL RESULTS}

Upon the onset of the irradiation, multiple new absorption features arose (Figure 1; Table 1). The majority of these features could be assigned to three discrete molecules: benzene, vinylacetylene, and its methylenecyclopropene isomer. The benzene molecule could be identified via its prominent $v_{19}$ ring stretching mode at $1480 \mathrm{~cm}^{-1}$, the $v_{13}+v_{16} \mathrm{C}-\mathrm{H}$ stretching at about $3095 \mathrm{~cm}^{-1}$, and the $v_{11} \mathrm{C}-\mathrm{H}$ bending mode between 671 and $690 \mathrm{~cm}^{-1}$. In D2-acetylene, the $v_{19}$ ring stretching mode shifts significantly to $1329 \mathrm{~cm}^{-1}$. Besides benzene, we also identified the vinylacetylene molecule via six carriers at $3280\left(v_{1}\right), 2976\left(v_{6}+v_{7}\right), 1241\left(2 v_{17}\right), 1599\left(v_{6}\right), 960\left(v_{14}\right)$, and $930 \mathrm{~cm}^{-1}\left(v_{15}\right)$. The formation of this molecule was confirmed in D2-acetylene via its $v_{5}, v_{14}+v_{15}$, and $v_{14}+v_{16}$ absorptions at 1967, 1875, and $1682 \mathrm{~cm}^{-1}$ and in ${ }^{13} \mathrm{C}_{2} \mathrm{H}_{2}$ through the $v_{14}, v_{13}, v_{9}$, and $v_{8}$ bands at 2070, 1552, 957, and $912 \mathrm{~cm}^{-1}$. Finally, the thermodynamically less stable methylenecyclopropene isomer of vinylacetylene was visible through its $v_{1}$ and $v_{2}$ carriers at 1764 and $650 \mathrm{~cm}^{-1}$, respectively. This was confirmed in irradiated ${ }^{13} \mathrm{C}_{2} \mathrm{H}_{2}$ ices through its absorptions at 1711 and $648 \mathrm{~cm}^{-1}$ attributed to the $v_{14}$ and $v_{3}$ bands, respectively. The two most intense absorptions of the D4-methylenecyclopropene overlap with those from the D2acetylene ices. It is noteworthy that besides the carriers assigned to benzene, vinylacetylene, and methylenecyclopropene, a few absorptions could not be attributed to any of these molecules (Table 1). They could be assigned to cumulenic $(\mathrm{C}=\mathrm{C}=\mathrm{C})$ and vinylic $\mathrm{CH}=\mathrm{CH}_{2}$ functional groups. We also quantified the newly formed molecules at the end of the irradiation exposure via their column densities (Bennett et al. 2005). Here, the column density of vinylacetylene decreases with rising temperature from $3.5 \pm 0.5 \times 10^{15} \mathrm{~cm}^{-2}(10 \mathrm{~K})$ to $1.8 \pm$ $0.3 \times 10^{15} \mathrm{~cm}^{-2}(67 \mathrm{~K})$. This trend is also reflected in the column densities of its methylenecyclopropene isomer (1.9 \pm $0.2 \times 10^{14} \mathrm{~cm}^{-2}(10 \mathrm{~K})$ to $\left.1.3 \pm 0.2 \times 10^{14} \mathrm{~cm}^{-2}(67 \mathrm{~K})\right)$; the column density of the vinylacetylene molecule is enhanced by one order of magnitude compared to its thermodynamically less stable methylenecyclopropene isomer. For benzene, the column density almost doubles with rising temperature from $1.8 \pm 0.3 \times 10^{15} \mathrm{~cm}^{-2}(10 \mathrm{~K})$ via $2.7 \pm 0.3 \times 10^{15} \mathrm{~cm}^{-2}$ $(30 \mathrm{~K})$ and $3.0 \pm 0.3 \times 10^{15} \mathrm{~cm}^{-2}(50 \mathrm{~K})$ to $3.4 \pm 0.4 \times$ $10^{15} \mathrm{~cm}^{-2}(67 \mathrm{~K})$. We can also calculate the production rates of the molecules per eV absorbed energy. These data are important to link the laboratory experiments to Titan. Electron trajectory calculations depict that each $5 \mathrm{keV}$ electron transfers on average $1.1 \pm 0.1 \mathrm{keV}$ to the acetylene target (Hovington 


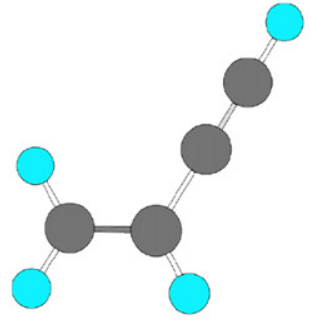

(1)

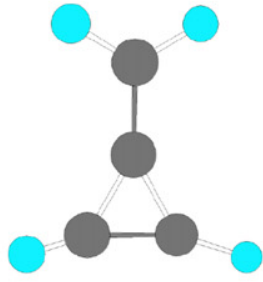

(2)

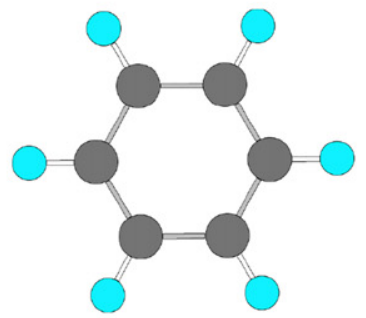

(3)

(a)
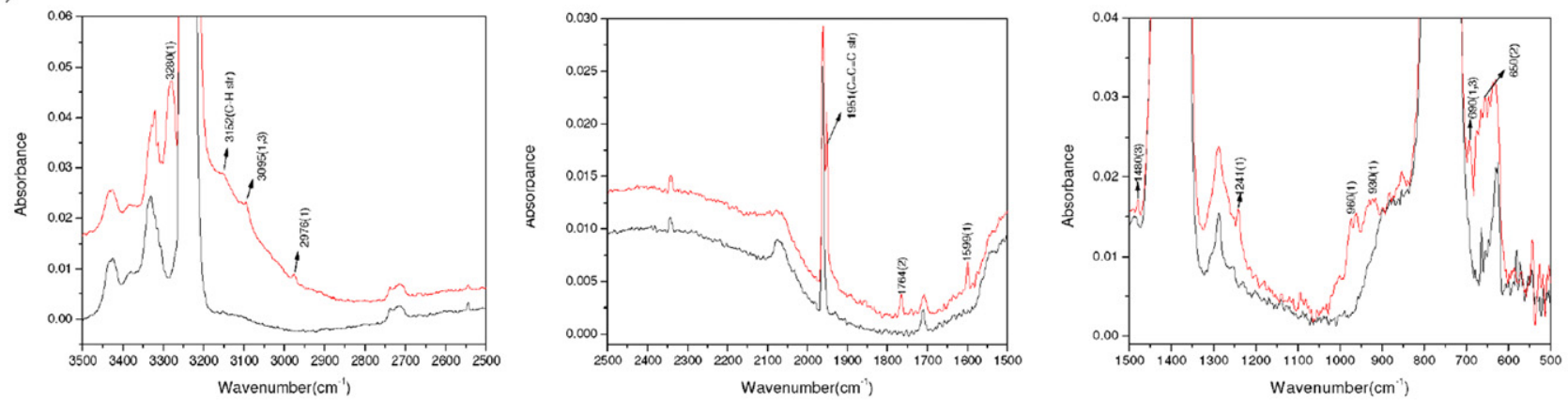

(b)
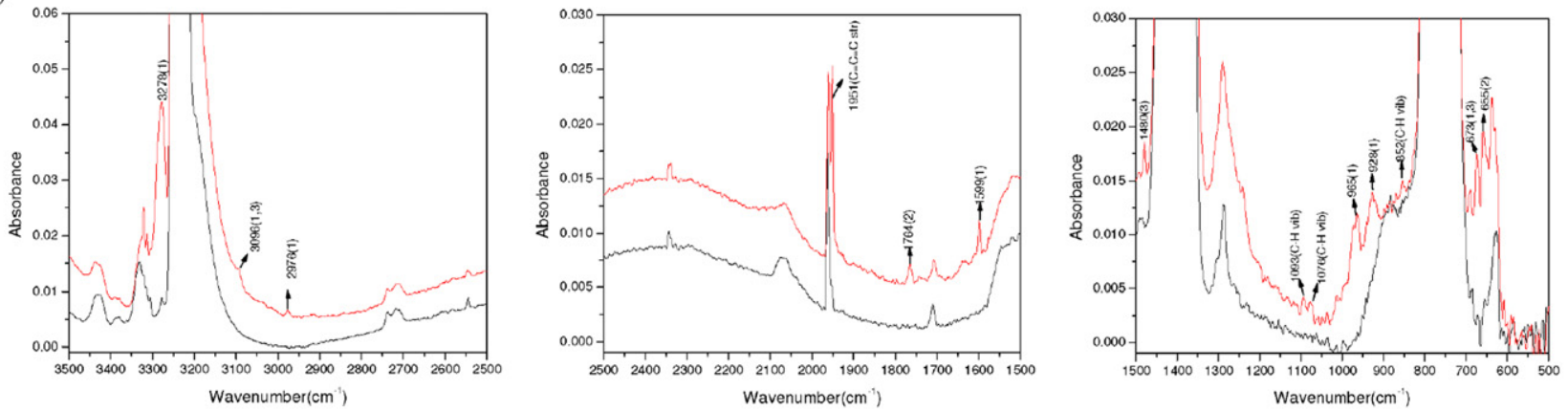

(c)
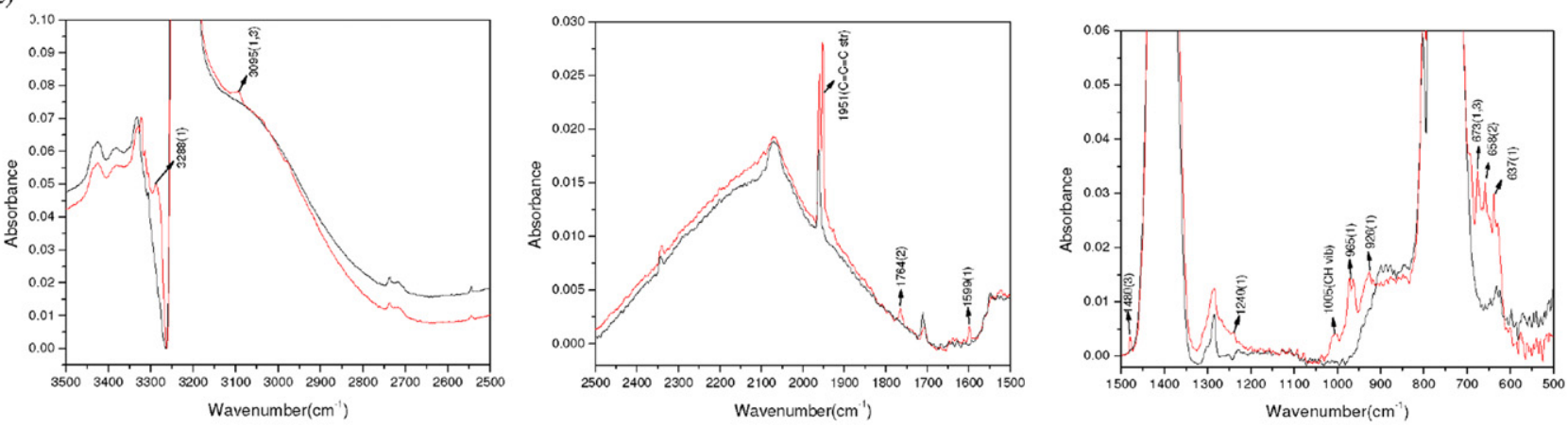

(d)
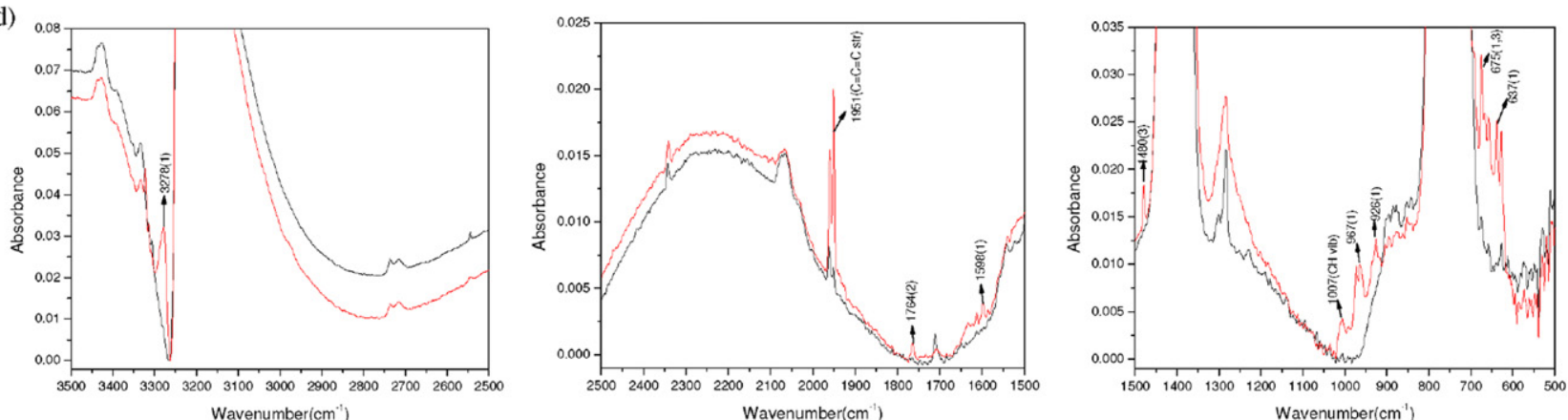

Figure 1. Infrared spectra of the acetylene samples recorded before (black line) and after (red line) the irradiation with energetic electrons at $10 \mathrm{~K}$ (a), $30 \mathrm{~K}$ (b), $50 \mathrm{~K}$ (c), and $67 \mathrm{~K}(\mathrm{~d})$. The assignments of the new absorptions are compiled in Table 1. The labels of the fundamentals, overtones, and combination bands belonging to acetylene are omitted for clarity. Molecules labeled as (1), (2), and (3) correspond to vinylacetylene $\left(\mathrm{C}_{4} \mathrm{H}_{4}\right)$, its methylenecyclopropene isomer $\left(\mathrm{C}_{4} \mathrm{H}_{4}\right)$, and benzene $\left(\mathrm{C}_{6} \mathrm{H}_{6}\right)$, respectively, whose molecular structures are depicted above.

(A color version of this figure is available in the online journal.) 
Table 1

Absorption Features Observed After the Irradiation of Acetylene Ices with Energetic Electrons at Different Temperatures

\begin{tabular}{|c|c|c|c|c|c|c|}
\hline $10 \mathrm{~K}$ & $30 \mathrm{~K}$ & $50 \mathrm{~K}$ & $67 \mathrm{~K}$ & Literature & Assignment & Carrier \\
\hline \multicolumn{7}{|c|}{ Absorption $\left(\mathrm{cm}^{-1}\right)$} \\
\hline 3280 & 3278 & 3280 & 3278 & $3287^{\mathrm{a}}$ & $v_{1} \mathrm{C}-\mathrm{H}$ stretch & Vinylacetylene \\
\hline 3152 & & & 3152 & & & as. $\mathrm{CH}_{2}$ stretch in $-\mathrm{CH}=\mathrm{CH}_{2}$ \\
\hline 3095 & 3095 & 3095 & 3095 & $3080^{\mathrm{b}}$ & $v_{13}+v_{16} \mathrm{C}-\mathrm{H}$ stretch & Benzene \\
\hline 2976 & 2976 & 2976 & 2976 & $2973^{\mathrm{a}}$ & $v_{6}+v_{7}$ & Vinylacetylene \\
\hline 1951 & 1951 & 1951 & 1951 & & & as. $\mathrm{C}=\mathrm{C}=\mathrm{C}$ stretch in $-\mathrm{HC}=\mathrm{C}=\mathrm{CH}_{2}$ \\
\hline 1764 & 1764 & 1764 & 1764 & $1770^{\mathrm{c}}$ & $v_{1}$ & Methylenecyclopropene \\
\hline 1599 & 1599 & 1599 & 1598 & $1600^{\mathrm{a}}$ & $v_{6}$ & Vinylacetylene \\
\hline 1480 & 1480 & 1480 & 1480 & $1486^{\mathrm{b}}$ & $v_{19}$ ring stretch & Benzene \\
\hline \multirow[t]{2}{*}{1241} & 1241 & 1240 & & $1245^{\mathrm{a}}$ & $2 \times v_{17}$ & Vinylacetylene \\
\hline & $1093 / 1076$ & 1005 & 1007 & & & Vinylacetylene \\
\hline 960 & 965 & 965 & 967 & $950^{\mathrm{a}}$ & $v_{14}$ & Vinylacetylene \\
\hline \multirow[t]{2}{*}{930} & 928 & 926 & 926 & $934^{\mathrm{a}}$ & $v_{15}$ & Vinylacetylene \\
\hline & 852 & & & & & Out-of-plane $\mathrm{CH}_{2}$ vib in $-\mathrm{CH}=\mathrm{CH}_{2}$ \\
\hline 690 & 671 & 673 & 675 & $673^{\mathrm{b}}$ & $v_{11} \mathrm{C}-\mathrm{H}$ bend & Benzene \\
\hline \multirow[t]{2}{*}{650} & 655 & 658 & & $664^{\mathrm{c}}$ & $v_{2}$ & Methylenecyclopropene \\
\hline & & 637 & 630 & $630^{\mathrm{a}}$ & $v_{11}$ & Vinylacetylene \\
\hline
\end{tabular}

Notes.

a Sheppard (1949).

b Marzocchi et al. (1970).

${ }^{\mathrm{c}}$ Billups et al. (1984).

et al. 2006). This Monte Carlo code utilizes the kinetic energy, the target composition (chemical composition, density), and the target thickness as input parameters and calculates, for instance, the energy loss of the energetic electron while passing through the solid state target. Accounting for the electron current and the irradiation time, $1.9 \pm 0.2 \times 10^{18} \mathrm{eV}$ are absorbed by the sample. Considering the thickness, surface area, molecular mass, and density of the acetylene samples, this energy is absorbed by $0.9 \pm 0.3 \times 10^{18}$ molecules, i.e., an averaged dose of $2.1 \pm 0.8 \mathrm{eV}$ per molecule. On average, benzene production rates of $3.2 \pm 0.3 \times 10^{-3}(10 \mathrm{~K}), 4.4 \pm 0.3 \times 10^{-3}(30 \mathrm{~K}), 5.1 \pm$ $0.3 \times 10^{-3}(50 \mathrm{~K})$, and $5.6 \pm 1.5 \times 10^{-3}(67 \mathrm{~K})$ molecules per $\mathrm{eV}$ have been derived.

The identification of these discrete molecules also gains support from the mass spectrometric data. Upon warming up the irradiated samples, acetylene molecules could be monitored via the $\mathrm{C}_{2} \mathrm{H}_{2}{ }^{+}$molecular ion at $m / z=26$ starting at $60-65 \mathrm{~K}$ (Figure 2). A quantitative integration of the ion current of acetylene during the sublimation and comparison with the nonirradiated, subliming sample suggests that about $13 \% \pm 2 \%$ of the acetylene molecules were destroyed by the irradiation. This value is invariant with the temperature of the irradiated target between $10 \mathrm{~K}$ and $67 \mathrm{~K}$. At higher temperatures of $105-108 \mathrm{~K}$, signal at $m / z=52\left(\mathrm{C}_{4} \mathrm{H}_{4}{ }^{+}\right)$and $m / z=50\left(\mathrm{C}_{4} \mathrm{H}_{2}{ }^{+}\right)$ emerged. In all experiments, the patterns of the ion currents at $m / z=50\left(\mathrm{C}_{4} \mathrm{H}_{2}{ }^{+}\right)$were identical to those recorded at $m / z=52\left(\mathrm{C}_{4} \mathrm{H}_{4}{ }^{+}\right)$. Therefore, signal at $m / z=52$ originated from dissociative ionization of the $\mathrm{C}_{4} \mathrm{H}_{4}$ neutral in the electron impact ionizer. Finally, at temperatures of about $130 \mathrm{~K}$, ion currents at $m / z=78\left(\mathrm{C}_{6} \mathrm{H}_{6}{ }^{+}\right)$became visible. The profiles at $m / z=78$ were distinct from those observed at $m / z=52$ and do not overlap with the latter. Therefore, signal at $m / z=$ 52 was not a fragment ion from $m / z=78$, but a distinct product species. Since neither $\mathrm{C}_{4} \mathrm{H}_{4}{ }^{+}$nor $\mathrm{C}_{6} \mathrm{H}_{6}{ }^{+}$ions were detected over the corresponding temperature regimes in the non-irradiated sample upon warming up, we can conclude that molecules of the formula $\mathrm{C}_{4} \mathrm{H}_{4}$ and $\mathrm{C}_{6} \mathrm{H}_{6}$ were formed during the radiation exposure. We should stress that we also observed the isotopically labeled counterparts in the control experiments of electron-irradiated deuterated and ${ }^{13} \mathrm{C}$-substituted acetylene samples at $m / z=56\left(\mathrm{C}_{4} \mathrm{D}_{4}{ }^{+} /{ }^{13} \mathrm{C}_{4} \mathrm{H}_{4}{ }^{+}\right)$and $m / z=84\left(\mathrm{C}_{6} \mathrm{D}_{6}{ }^{+} /\right.$ $\left.{ }^{13} \mathrm{C}_{6} \mathrm{H}_{6}{ }^{+}\right)$. To summarize, the mass spectrometric data correlate with the infrared spectroscopic assignment of benzene as well as vinylacetylene and its methylenecyclopropene isomer. Here, these subliming molecules result in mass spectrometric ion counts of their molecular ions at $m / z=78\left(\mathrm{C}_{6} \mathrm{H}_{6}{ }^{+}\right)$as well as $m / z=52\left(\mathrm{C}_{4} \mathrm{H}_{4}{ }^{+}\right)$. Note that the mass spectrometric signatures alone would be no evidence of, for instance, benzene, since multiple structural isomers can account for signal at $\mathrm{m} / z=$ 78. Therefore, only the combination of mass spectroscopy and infrared spectroscopy together with isotopically labeled reactants allows us to assign the discrete molecular reaction products unambiguously.

We can also estimate the conversion efficiency of acetylene into the polymeric residues and discrete molecules (Figure 2; Tables 2 and 3). Taking the column densities of the newly formed vinylacetylene, methylenecyclopropene, and benzene molecules and accounting for the irradiated sample area of $3.1 \pm 0.2 \mathrm{~cm}^{2}$ and the fact that vinylacetylene/ methylenecyclopropene and benzene contain formally two and three acetylene units, respectively, we compute the number of acetylene molecules incorporated into the products. We can then sum up these data to extract the total number of acetylene molecules converted into discrete molecules. Our calculations suggest that on average, $4.3 \pm 0.8 \times 10^{16}$ acetylene molecules are converted in total into vinylacetylene, methylenecyclopropene, and benzene. The mass spectrometric results suggested that an overall of $13 \% \pm 2 \%$ of the acetylene, i.e., $1.9 \pm 0.2 \times$ $10^{17}$ molecules, were integrated into molecular products (vinylacetylene, methylenecyclopropene, benzene) and the polymeric residue. This allows an estimate that $81 \% \pm 5 \%$ of the acetylene molecules were converted to solid residues and $19 \% \pm$ $5 \%$ to vinylacetylene, methylenecyclopropene, and benzene; those values are temperature independent within the range of $10 \mathrm{~K}-67 \mathrm{~K}$. The calculated production rates of the newly formed molecules in units of molecules per in $\mathrm{eV}$ absorbed are compiled 


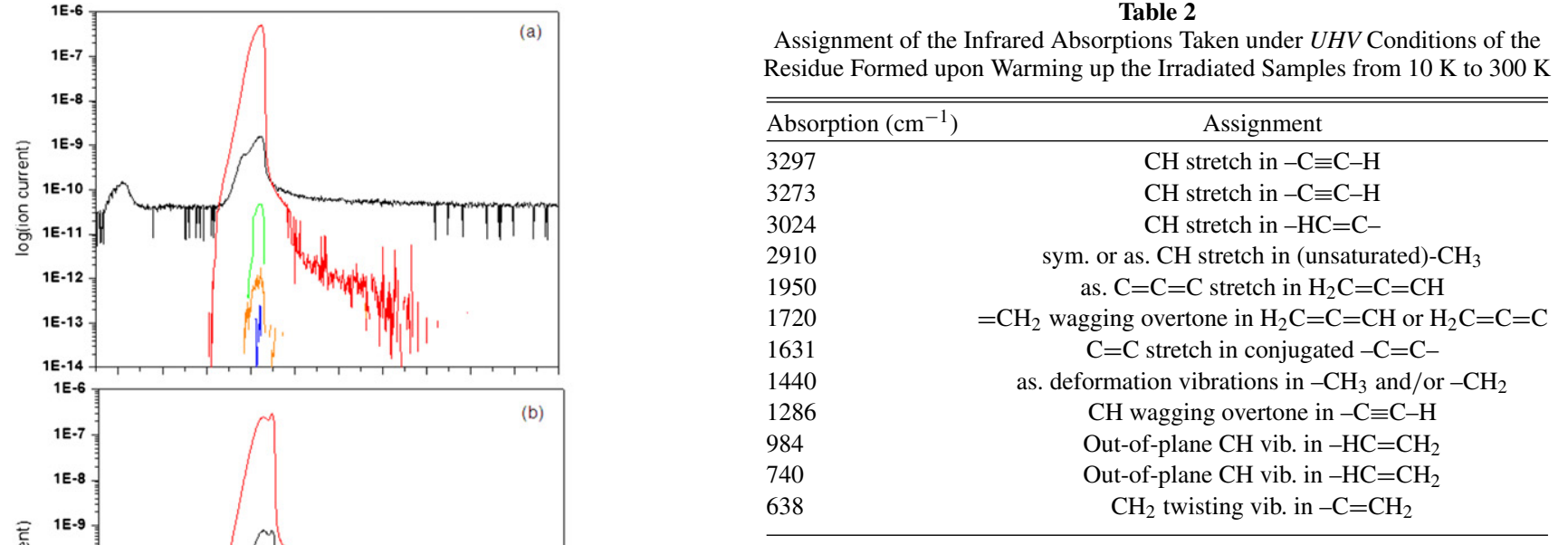

in Table 3. On average, $9.5 \pm 2.0 \times 10^{-2}$ acetylene molecules per $\mathrm{eV}$ are converted into a solid polymer over the temperature range investigated.

To get a picture of the chemical composition of the solid residues (Figure 3), particularly with respect to the potential existence of aromatic molecules such as complex PAHs, we analyzed the residues of the samples utilizing the laser desorption/ ionization (LDI) technique using either an infrared laser (carbon dioxide laser; $10.6 \mu \mathrm{m}$ wavelength) or a nitrogen laser (337 nm). The infrared photons employed in the experiments can only liberate free molecules; the resonantly enhanced multi-photon ionization scheme sensitively and selectively ionizes PAH species (Clemett \& Zare 1997) However, none of the samples subjected to infrared photons yielded any measurable signal; this means that free PAH molecules constitute at most an insignificant fraction of the samples $(<100 \mathrm{ppb})$ (Elsila et al. 2004). This correlates nicely with the in situ mass spectrometric analysis during the sublimation of the electron-irradiated samples, which manifested that benzene $(m / z=78)$ is the heaviest, discrete aromatic product formed. However, the more energetic ultraviolet photons are able to fragment the polymer bonds in the residues, thus releasing material into the gas phase, a small percentage of which is ionized (Murgasova \& Hercules 2003). Results from this analysis are presented in Figure 4. The spectra show a characteristic shape that resembles the LDI mass spectra of highly polydisperse polymers possibly formed via cross linking of the acetylene monomers (Chen et al. 2001) and continue to show mass peaks at every nominal mass out to just above $900 \mathrm{amu}$ (not pictured). Given the fragmentary nature of LDI, this likely represents a minimum estimate for the upper-limit size of the constituent polymer residues. The low-mass region of the spectra is dominated by peak envelopes whose central masses are spaced 14 amu apart. A closer look at these peak envelopes reveals a complex chemistry. The envelopes contain peaks at every nominal mass and span at least $14 \mathrm{amu}$. The prominence of peaks at spacings of 2 amu below a nominal alkane mass is typical of the presence of alkenes $\left(-\mathrm{H}_{2}\right)$.

\section{THEORETICAL RESULTS}

Our calculation suggests that two singlet ground-state acetylene molecules do not react with each other due to an insurmountable entrance barrier to reaction of $140 \mathrm{~kJ} \mathrm{~mol}^{-1}$ which is typical for closed shell reactants. This finding can also be transferred to the solid state; here, two ground-state acetylene molecules do not react with each other since at low temperatures
Figure 2. Ion currents of $m / z=2\left(\mathrm{H}_{2}^{+}\right.$; black), $26\left(\mathrm{C}_{2} \mathrm{H}_{2}^{+}\right.$, red $), 50\left(\mathrm{C}_{4} \mathrm{H}_{2}^{+}\right.$, blue), $52\left(\mathrm{C}_{4} \mathrm{H}_{4}^{+}\right.$, green $)$, and $78\left(\mathrm{C}_{6} \mathrm{H}_{6}^{+}\right.$, orange $)$obtained in the warming up phase of the acetylene samples irradiated at $10 \mathrm{~K}(\mathrm{~b}), 30 \mathrm{~K}(\mathrm{c}), 50 \mathrm{~K}(\mathrm{~d})$, and $67 \mathrm{~K}(\mathrm{e})$. Ion currents for the blank experiments (a) were also recorded; the ion profiles for $m / z=2$ in the temperature range of $10 \mathrm{~K}-20 \mathrm{~K}$ originate from molecular hydrogen subliming from copper cold head target upon warming up.

(A color version of this figure is available in the online journal.) 

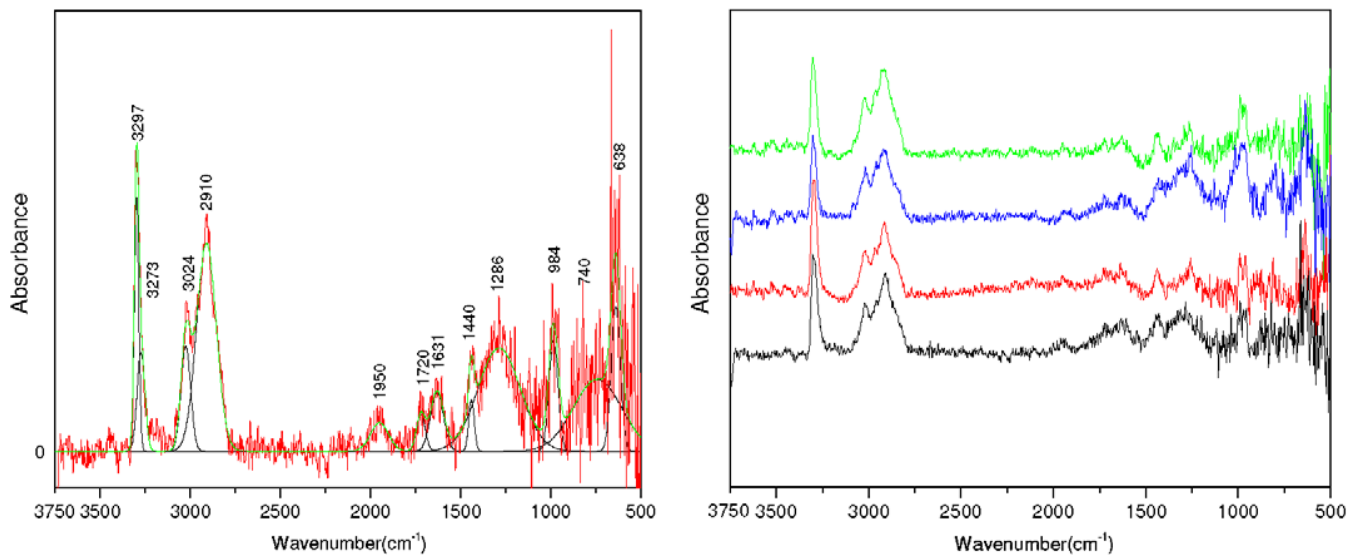

Figure 3. Infrared spectra of the residues obtained after warming up the irradiated sample to $300 \mathrm{~K}$ for irradiations conducted at $10 \mathrm{~K}$ (green; a), $30 \mathrm{~K}$ (blue; b), $50 \mathrm{~K}$ (red; c), and $67 \mathrm{~K}$ (black; d). The peak positions are compiled in Table 2.

(A color version of this figure is available in the online journal.)

Table 3

Numbers of Acetylene Molecules Accounting for the Vinylacetylene, Methylenecyclopropene, and Benzene Molecules Produced at the End of the Irradiation of Acetylene Ices with Energetic Electrons together with the Sum

\begin{tabular}{ccccc}
\hline \hline$T$ & Vinylacetylene & Methylenecyclopropene & Benzene & Sum \\
\hline $10 \mathrm{~K}$ & $2.2 \pm 0.3 \times 10^{16}$ & $9.9 \pm 1.0 \times 10^{14}$ & $1.7 \pm 0.3 \times 10^{16}$ & $4.0 \pm 0.6 \times 10^{16}$ \\
& $\left(5.8 \pm 1.5 \times 10^{-3}\right)$ & $\left(2.6 \pm 0.3 \times 10^{-4}\right)$ & $\left(3.1 \pm 1.0 \times 10^{-3}\right)$ & $4.8 \pm 0.9 \times 10^{16}$ \\
\hline $30 \mathrm{~K}$ & $2.2 \pm 0.3 \times 10^{16}$ & $10.5 \pm 1.0 \times 10^{14}$ & $2.6 \pm 0.5 \times 10^{16}$ & $\left(4.4 \pm 1.2 \times 10^{-3}\right)$ \\
& $\left(5.8 \pm 1.5 \times 10^{-3}\right)$ & $\left(2.7 \pm 0.5 \times 10^{-4}\right)$ & $2.9 \pm 0.5 \times 10^{16}$ & $4.3 \pm 0.9 \times 10^{16}$ \\
\hline $50 \mathrm{~K}$ & $1.4 \pm 0.3 \times 10^{16}$ & $8.7 \pm 0.9 \times 10^{14}$ & $\left(5.1 \pm 1.5 \times 10^{-3}\right)$ & \\
& $\left(3.6 \pm 0.9 \times 10^{-3}\right)$ & $\left(2.2 \pm 0.5 \times 10^{-4}\right)$ & $3.2 \pm 0.7 \times 10^{16}$ & $4.4 \pm 1.0 \times 10^{16}$ \\
$67 \mathrm{~K}$ & $1.2 \pm 0.3 \times 10^{16}$ & $6.8 \pm 0.9 \times 10^{14}$ & $\left(5.6 \pm 1.5 \times 10^{-3}\right)$ & \\
& $\left(3.1 \pm 0.9 \times 10^{-3}\right)$ & $\left(1.9 \pm 0.3 \times 10^{-4}\right)$ & & \\
\hline
\end{tabular}

Note.

${ }^{a}$ The numbers in parentheses present the production rates of the newly formed molecules in units of molecules per eV absorbed.
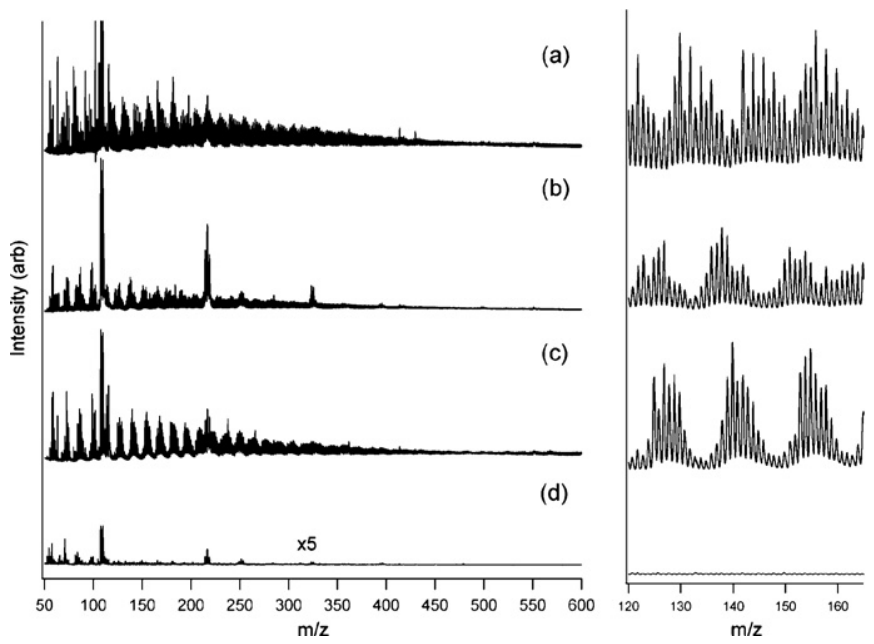

Figure 4. Laser desorption mass spectra (LDMS) of the room temperature residue from (a) irradiated acetylene ice, (b) irradiated $\mathrm{D}_{2}$-acetylene ice, (c) irradiated ${ }^{13} \mathrm{C}$-acetlyene ice, and (d) a procedural blank. The inset at right zooms in on the low-mass peak envelopes highlighting the shifts due to isotopic differences among the three samples $(\mathrm{m} / z=$ mass-to-charge ratio).

in the ice, the entrance barrier to reaction cannot be overcome. However, an acetylene molecule in an excited electronic state is expected to be much more reactive. We have chosen an acetylene molecule in its first excited triplet state, ${ }^{3} B_{2}$, as a model to investigate the reactivity of electronically excited acetylene with the ground-state acetylene counterparts. Our calculations show that $\mathrm{C}_{2} \mathrm{H}_{2}\left(\mathrm{a}^{3} B_{2}\right)$ can indeed easily react with ground-state acetylene via a mild barrier of $4 \mathrm{~kJ} \mathrm{~mol}^{-1}$ to form a trans-CHCHCHCH structure [1] (Figure 5). The latter can undergo trans-cis isomerization to [2]. Triplet cis-CHCHCHCH [2] can now react with a second ground-state acetylene molecule to form [3]. The inherent barrier to addition of $20 \mathrm{~kJ} \mathrm{~mol}^{-1}$ lies below the energy of the separated reactants, $\mathrm{C}_{2} \mathrm{H}_{2}\left(\mathrm{a}^{3} B_{2}\right)+2 \mathrm{C}_{2} \mathrm{H}_{2}\left(\mathrm{X}^{1} \Sigma_{\mathrm{g}}{ }^{+}\right)$, and so the triplet cis-CHCHCHCH [2] $+\mathrm{C}_{2} \mathrm{H}_{2}\left(\mathrm{X}^{1} \Sigma_{\mathrm{g}}{ }^{+}\right)$reaction can be possible even at low temperatures if [2] keeps enough energy acquired from the initial reactive encounter, $\mathrm{C}_{2} \mathrm{H}_{2}\left(\mathrm{a}^{3} B_{2}\right)+$ $\mathrm{C}_{2} \mathrm{H}_{2}\left(\mathrm{X}^{1} \Sigma_{\mathrm{g}}{ }^{+}\right)$. The triplet intermediate can easily rearrange to [4] followed by ring closure to triplet benzene [5]. Again, we shall stress that these calculations are done to test if excited state acetylene molecules such as the ${ }^{3} B_{2}$ state can react with a ground-state acetylene molecule; of course, the electrons can also excite the acetylene molecule to excited state higher than the ${ }^{3} B_{2}$ level investigated here. Also, in the solid state, processes such as multi-body reactions, stabilization of internally and/or electronically excited intermediates, and the geometrical orientation of the neighboring molecules can significantly influence the outcome of the reaction. Therefore, the calculations should be taken as guidance for one possible reaction pathway highlighting excited state reactions. In the acetylene matrix, for instance, the ${ }^{3} B_{2}$ state could be accessible via an energy transfer from the impinging electrons to an acetylene molecule. If the excited molecule holds the correct geometry to react with a ground-state acetylene molecule, reaction to 


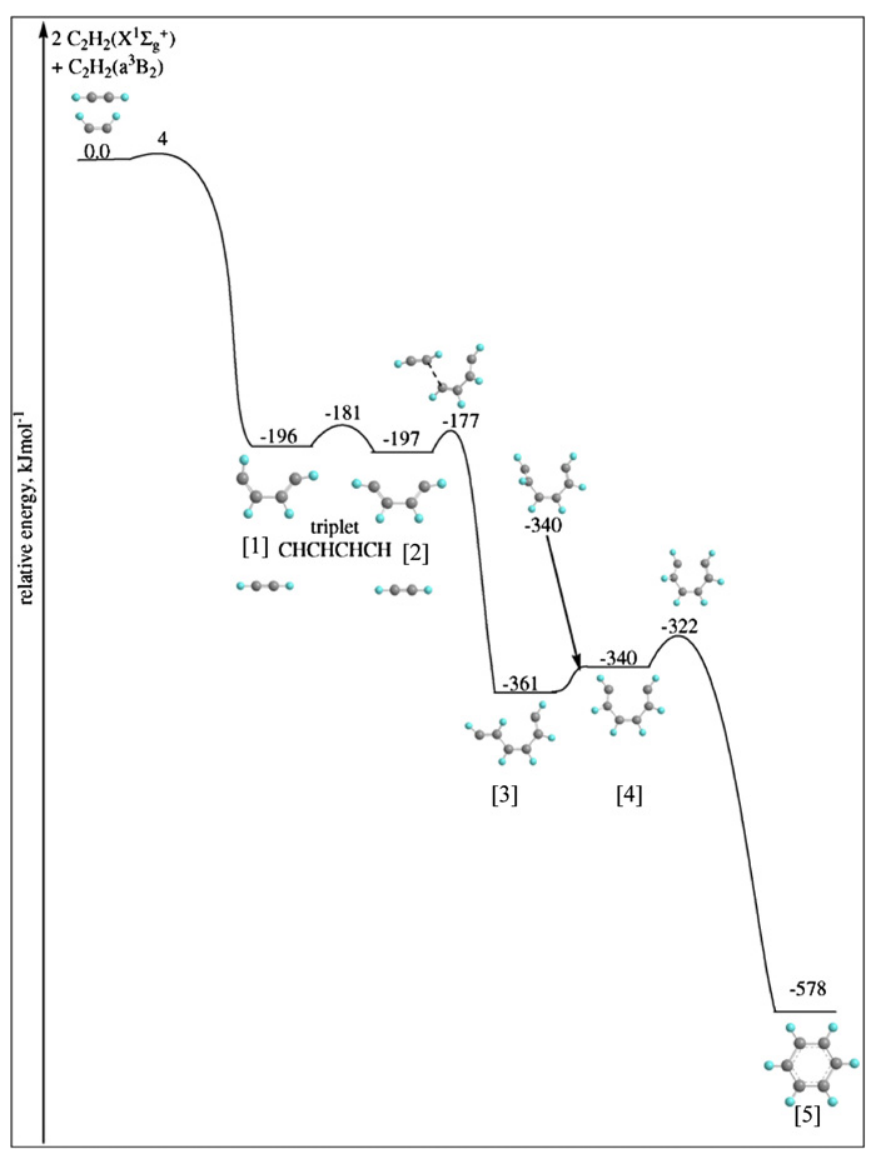

Figure 5. Part of the triplet $\mathrm{C}_{6} \mathrm{H}_{6}$ potential energy surface involved in the formation of triplet benzene via excited state acetylene reactions. Energies are given in $\mathrm{kJ} \mathrm{mol}^{-1}$. With the exception of the first addition step, all energies of the transition states and intermediates are below the energies of the separated reactants. Triplet benzene can undergo intersystem crossing to the singlet surface.

(A color version of this figure is available in the online journal.)

trans-CHCHCHCH [1] might be feasible if the entrance barrier can be overcome by, for instance, vibrational energy in one of the reactant molecules. Similar to electronic excitation, the high-energy electrons can produce a vibrationally excited acetylene molecule. Upon reaction with a neighboring acetylene molecule, trans- $\mathrm{CHCHCHCH}$ could react to-via [3] and [4] - triplet benzene, which can undergo intersystem crossing to the singlet manifold; note that singlet benzene is $372 \mathrm{~kJ} \mathrm{~mol}^{-1}$ lower in energy than triplet benzene. Other pathways can exist as well. For example, in the related acetylene-carbon monoxide system, triplet acetylene or triplet carbon monoxide was found to react with singlet carbon monoxide or singlet acetylene to form the cyclopropenone molecule, $\mathrm{c}-\mathrm{C}_{3} \mathrm{H}_{2} \mathrm{O}$, within one step (Zhou et al. 2008). Likewise, an acetylene molecule in neighborhood to a carbon monoxide molecule was found to undergo unimolecular decomposition to an ethynyl radical (HCC) plus atomic hydrogen; the latter added to the carbon monoxide molecule leading the formyl (HCO) radical which then recombined with the ethynyl radical to the propynal ( $\mathrm{HCCCHO}$ ) isomer. In the acetylene system, an acetylene molecule may also fragment to an ethynyl radical (HCC) plus hydrogen atom. The atomic hydrogen can add to a second acetylene molecule forming the vinyl radical $\left(\mathrm{C}_{2} \mathrm{H}_{3}\right)$ which in turn recombines without barrier with a neighboring ethynyl radical to yield vinylacetylene as detected in our experiments. Also, an electronically excited acetylene molecule may isomerize to its vinylidene isomer $\left(\mathrm{H}_{2} \mathrm{CC}\right)$, which in turn adds to the carbon-carbon triple bond of a second, neighboring acetylene molecule in its correct geometry yielding the experimentally observed methylenecyclopropene isomer. The lack of "free" ethynyl radicals could also explain the failed detection of diacetylene $(\mathrm{HCCCCH})$ - a likely recombination product of two ethynyl radicals. Note that, in analogy with the propynal and cyclopropenone profiles in the acetylene-carbon monoxide system, the concentration profiles of vinylacetylene and methylenecyclopropene could be fit with pseudo-first-order kinetics (Figure 6). We would like to stress that the column density for benzene could also be fit with pseudo-first-order kinetics, despite a proposed, multi-step reaction sequence based on the out potential energy surface. Nevertheless, if all reactions, with the exception of the initial addition to form [1], are really fast, a pseudo-first-order fit is reasonable.

\section{ASTROPHYSICAL IMPLICATIONS}

Having established that energetic electrons as generated in the track of cosmic-ray particles can form benzene molecules upon interaction with low-temperature acetylene ices via nonequilibrium, excited state chemistry, we transfer now our findings from the laboratory to the "real" setting in our solar system: Titan. This connection is crucial since the overall global effects of the cosmic-ray processing of Titan's surface with respect to Titan's benzene budget can be evaluated. Here, the total amount of benzene produced on the surface is given by

$$
M=\phi_{\mathrm{CR}} \times Y \times F_{o} \times F_{a} \times \tau
$$

with the cosmic-ray flux on Titan's surface $\left(\phi_{\mathrm{CR}}=\right.$ $10^{9} \mathrm{eV} \mathrm{cm}^{-2} \mathrm{~s}^{-1}$ ) as derived from Molina-Cuberosa et al. (1999), the yield of benzene from solid acetylene as extracted from our laboratory experiments $\left(Y=5.6 \times 10^{-3} \mathrm{eV}^{-1}\right)$, the fraction of the surface of Titan covered by organics $\left(F_{o}\right)$, fraction of organics that is acetylene $\left(F_{a}\right)$, and the time for turnover of the surface by geological processes $(\tau)$. The last three factors justify some discussion. Conservatively, we take $F_{o}=0.2$ and $F_{a}=$ 0.2 as determined by the percentage of the surface surveyed that covers organic species (Lorenz et al. 2008) and fraction of acetylene of precipitable molecules (Yung et al. 1984), respectively. The surface of Titan is relatively young; estimates range from $2 \times 10^{6}$ to $10^{9}$ years (Lunine et al. 2005; Jaumann $\&$ Neukum 2009). Taking the lower value of $2 \times 10^{6}$ years, we estimate the total amount of benzene produced on the surface to be $M=1.4 \times 10^{19}$ molecules $\mathrm{cm}^{-2}$. The maximum rate of benzene formation via cosmic-ray exposure is $3.4 \times$ $10^{-17} \mathrm{~g} \mathrm{~cm}^{-2} \mathrm{~s}^{-1}$; this is small compared to the atmospheric production rate of $4 \times 10^{-14} \mathrm{~g} \mathrm{~cm}^{-2} \mathrm{~s}^{-1}$. However, this rate is significant compared to the modeled precipitation rate after considering chemical loss in the atmosphere of $10^{-17} \mathrm{~g} \mathrm{~cm}^{-2} \mathrm{~s}^{-1}$. Note that the maximum production rate of benzene by cosmic rays is globally averaged; the value can be as high as $7 \times$ $10^{-16} \mathrm{~g} \mathrm{~cm}^{-2} \mathrm{~s}^{-1}$ considering Molina-Cuberosa et al.'s cosmicray flux at locations where $F_{o}$ is close to unity. Based on these considerations, our studies suggest that cosmic-ray-mediated benzene formation on Titan's surface could be the dominant source of benzene in those regions of the surface which have a high surface coverage of acetylene. Most importantly, Titan's surface has the ability to sequester, i.e., to build up large inventories of the newly formed benzene molecules over the geologic timescale. Upon impact by the Huygens lander, a fraction of the benzene in the soil could have sublimed into the atmosphere due 

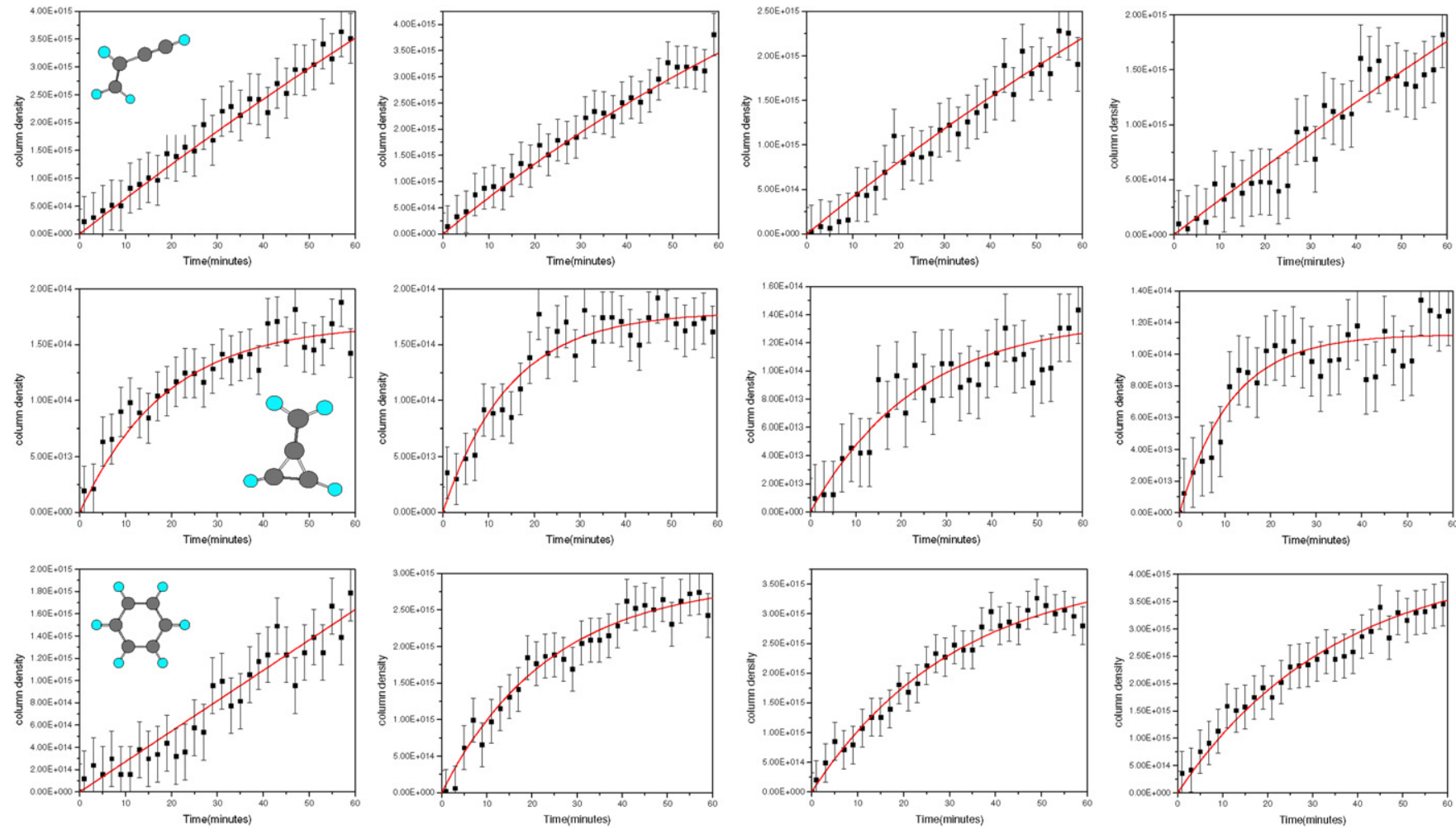

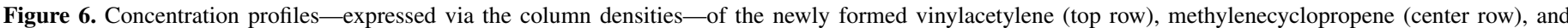

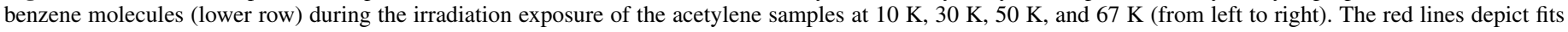
utilizing (pseudo) first-order kinetics.

(A color version of this figure is available in the online journal.)

to the elevated temperature of the Huygens lander compared to Titan's surface temperature of $94 \mathrm{~K}$. This aromatic component together with the polymeric residue formed in the laboratory experiments could resemble a potential source of organic material contributing to the makeup of Titan's organic sand dunes. To summarize, our laboratory simulation experiments identified the benzene molecule $\left(\mathrm{C}_{6} \mathrm{H}_{6}\right)$ as a product during the irradiation of acetylene ices $\left(\mathrm{C}_{2} \mathrm{H}_{2}\right)$ at low temperatures with energetic electrons as formed in the track of energetic cosmic-ray particles penetrating Titan's atmosphere down to its surface. The overall reaction transforms formally three acetylene molecules into one benzene molecule (Equation (2)). Supported by electronic structure calculations, the reaction mechanism involves nonequilibrium chemistry on excited state surfaces. On the triplet surface, for instance, a likely reaction pathway could be that triplet acetylene reacted with ground-state acetylene to a triplet $\mathrm{C}_{4} \mathrm{H}_{4}$ intermediate, which in turn reacted with a neighboring acetylene molecule to triplet benzene followed by intersystem crossing to the singlet manifold. A transfer of our findings to Titan suggests that cosmic-ray-mediated benzene formation on Titan's surface could be the dominant source of benzene, i.e., a factor of at least 100 higher compared to previously modeled precipitation rates, in those regions of the surface which have a high surface coverage of acetylene. The exact surface coverage of acetylene and the variation with longitude and latitude shall be a subject of future spectroscopic and/or in situ investigations of Titan:

$$
3 \mathrm{C}_{2} \mathrm{H}_{2} \rightarrow \mathrm{C}_{6} \mathrm{H}_{6}
$$

The experimental work was supported by the Chemistry Division of the US National Science Foundation within the framework of the Collaborative Research in Chemistry (CRC) Program (NSF-CRC CHE-0627854; LZ, WZ, RIK, AMM). We are very much indebted to Matthew R. Hammond and Richard N. Zare, Department of Chemistry, Stanford University, Stanford, CA, for the LDI measurements. One of us (Y.L.Y.) thanks T. Orlando (Georgia Tech) for valuable discussion. Y.L.Y. is supported by NASA grant NASA5-13296 to the California Institute of Technology. M.C.L. is supported by NSC grant 982111-M-001-014-MY3 to Academia Sinica. We thank Tobias Owen (Institute for Astronomy, University of Hawaii) and Roger Yelle (LPI, Arizona) for stimulating discussions and valuable comments on this manuscript. Special thanks are due to Keran O'Brien (Northern Arizona University) for sharing details on the calculations on the cosmic-ray exposure on Titan's surface.

\section{REFERENCES}

Becke, A. D. 1993, J. Chem. Phys., 98, 5648

Bennett, C. J., Jamieson, C. S., Osamura, Y., \& Kaiser, R. I. 2005, ApJ, 624, 1097

Billups, W. E., Lin, L.-J., \& Casserly, E. W. 1984, J. Am. Chem. Soc., 106, 3698

Chen, R., Yalcin, T., Wallace, W. E., Guttman, C. M., \& Li, L. 2001, J. Am. Soc. Mass Spectrom., 12, 1186

Clemett, S. J., \& Zare, R. N. 1997, in Molecules in Astrophysics: Probes and Processes, ed. E. F. V. Dishoeck (Leiden, The Netherlands: Kluwer), 305

Coustenis, A., \& Hirtzig, M. 2009, A\&A, 9, 249

Coustenis, A., Salama, A., Schulz, B., Ott, S., Lellouch, E., Encrenaz, T.h., Gautier, D., \& Feuchtgruber, H. 2003, Icarus, 161, 383

Coustenis, A., et al. 2007, Icarus, 189, 35

Elsila, J. E., De Leon, N. P., \& Zare, R. N. 2004, Anal. Chem., 76, 2430

Flasar, F. M. 2006, Science, 313, 1582

Griffith, C. A., Hall, J. L, \& Geballe, T. R. 2000, Science, 290, 509

Hovington, P., Drouin, D., Gauvin, R., Joy, D. C., \& Evans, N. 2006, Scanning, 19,29

Imanaka, H., et al. 2004, Icarus, 168, 344 
Jaumann, R., \& Neukum, G. 2009, The Surface Age of Titan, 40th Lunar and Planetary Institute Science Conference Abstracts, 1641

Lavvas, P. P., Coustenis, A., \& Vardavas, I. M. 2008, Planet. Space Sci., 56, 27

Lebonnois, S. 2005, Planet. Space Sci., 53, 486

Lee, C., Yang, W., \& Parr, R. G. 1988, Phys. Rev., B 37, 785

Liang, M. C., Yung, Y. L., \& Shemansky, D. E. 2007, ApJ, 661, L199

Lorenz, R. D., et al. 2008, Geophys. Res. Lett., 35, L02206/1

Lunine, J. I., \& Lorenz, R. D. 2009, Annu. Rev. Earth Planet. Sci., 37, 299

Lunine, J., Artemieva, N. A., Lorenz, R. D., \& Flamini, E. 2005, Numerical Modeling of Impact Cratering on Titan with Implications for the Age of Titan's Surface, 36th Annual Lunar and Planetary Science Conference Abstracts, 1504

Marzocchi, M. P., Bonadeo, H., \& Taddei, G. 1970, J. Chem. Phys., 53, 867

Molina-Cuberosa, G. J., et al. 1999, Planet. Space Sci., 47, 1347

Murgasova, R., \& Hercules, D. M. 2003, Int. J. Mass Spectrom., 226, 151

Niemann, H. B., et al. 2005, Nature, 438, 779
Pople, J. A., Head-Gordon, M., \& Raghavachari, K. 1987, J. Chem. Phys., 87, 5968

Purvis, G. D., \& Bartlett, R. J. 1982, J. Chem. Phys., 76, 1910, 76,

Raulin, F. 2008, Nature, 454, 587

Sagan, C., \& Thompson, W. R. 1984, Icarus, 59, 133

Scuseria, G. E., Janssen, C. L., \& Schaefer, H. F., III 1988, J. Chem. Phys., 89, 7382

Scuseria, G. E., \& Schaefer, H. F., III 1989, J. Chem. Phys., 90, 3700

Sheppard, N. 1949, J. Chem. Phys., 17, 74

Vuitton, V, Yelle, R. V., \& Cui, J. 2008, J. Geophys. Res.-Planets, 113, E05007/ 1

Wilson, E. H., \& Atreya, S. K. 2004, J. Geophys. Res.-Planets, 109, 20

Wilson, E. H., Atreya, S. K., \& Coustenis, A. 2003, J. Geophys. Res.-Planets, $108,8 / 1$

Yung, Y. L., Allen, M., \& Pinto, J. P. 1984, ApJS, 55, 465

Zhou, L., et al. 2008, ApJ, 686, 1493 